Article

\title{
Investigation of Second-Harmonic Generation and Molecular Orientation in Electrostatically Self-Assembled Thin Films
}

\section{Liangmin Zhang ${ }^{1, *}$ and Deliang Cui ${ }^{2}$}

1 Arkansas Center for Laser Applications and Science, Department of Chemistry and Physics, Arkansas State University, State University, Jonesboro, AR 72467, USA

2 National Laboratory of Crystal Materials, Shandong University, Jinan, Shandong 250100, China; E-Mail: cuidl@sdu.edu.cn

* Author to whom correspondence should be addressed; E-Mail: lzhang@astate.edu.

Received: 1 July 2011 / Accepted: 16 August 2011 / Published: 18 August 2011

\begin{abstract}
We report the observation and measurement of second-harmonic generation in self-assembled ultra thin film nonlinear optical materials using a femtosecond high repetition rate laser system. Second-harmonic intensity, as a function of the incident angle in these films, has been measured using incident p-polarized and s-polarized optical beam components. The second-order nonlinear optical susceptibilities of the thin films have also been determined. Using a curve fitting method and a crystal reference material, we have obtained second-order susceptibilities $\chi_{333}=6.17 \pm 0.18 \mathrm{pm} / \mathrm{V}$ and $\chi_{311}=0.68 \pm 0.02 \mathrm{pm} / \mathrm{V}$ at a fundamental wavelength of $1,200 \mathrm{~nm}$. Based on linear molecular model approximation, we have also used the fitted data to investigate the average orientation distribution of the chromophore dipoles in the self-assembled film. The result indicates that the average tilt angle of the chromophore dipoles away from the substrate normal line is $25.2^{\circ} \pm 0.8^{\circ}$.
\end{abstract}

Keywords: second-harmonic generation; second-order susceptibility; self-assembly; molecular orientation

\section{Introduction}

Recent interest in conjugated organic molecular and polymeric materials has been stimulated in part due to their intrinsic large optical nonlinearities. The strong second-harmonic (SH) generation in organic molecules originates from the fact that they possess electron donor and acceptor groups 
attached to a $\pi$-conjugated system. Since the first external electric field poled second order nonlinear optical (NLO) polymers were reported in 1982 [1,2], intensive studies have led to the development of commercial polymer guided-wave nonlinear optical devices [3]. Most of this work has concentrated on the development of spin-coated thin film materials. In order to possess nonzero even-order nonlinear electric susceptibilities, a material must lack a center of inversion at the macroscopic level. Therefore, a poling electric field must be applied to such spin-coated film materials to align the chromophores and remove the center of inversion [4-7]. This approach exhibits several difficulties. First, the field-induced polar ordering of guest chromophores is only temporarily frozen in, by cooling the field-oriented system below the glass transition temperature of the host polymer. Second, using the spin-coating technique it is generally difficult to obtain uniform and homogeneous films. Furthermore, the NLO response of poled polymers undergoes a spontaneously decay over time. Due to the thermal and temporal-dependent relaxation of chromophore alignment in conventional electric field-poled guest-host nonlinear optical polymer materials, the development of alternative techniques to form such materials without the need for electric field poling has been investigated. These techniques include the electrostatically self-assembly method by means of alternate adsorption of polycation and polyanion to build multilayer thin films on solid substrate [8,9], fabricating NLO superlattices by self-assembling via covalent linking [10], and the Langmuir method to prepare monolayers at the air-water interface [11], which provide new possibilities to fabricate NLO thin films without poling by an external electric field.

\section{Sample Preparation}

Electrostatically self-assembled (ESA) processes have recently been developed, which allow detailed structural control at the molecular level with ease of manufacturing [8]. The ESA method involves the alternate dipping of a charged substrate into aqueous solutions of oppositely charged ions at room temperature. In this method, the crucial feature is the alternate adsorption of positively and negatively charged ions to build up a thin film with the layer-by-layer procedure. When a polyelectrolyte adsorbs onto an oppositely charged surface, charge reversal on the surface can typically occur, this then facilitates the adsorption of the next layer of oppositely charged polymer or particles. In principle, films with an arbitrary number of bilayers can be fabricated by repeating the adsorption process. The ESA technique has been proven to provide a noncentrosymmetric arrangement of NLO chromophores in a multilayered thin film using a number of commercially available and synthesized ionic polymer dyes. The ESA layer-by-layer molecular processing approach has been suggested as a possible method to achieve the noncentrosymmetric structure without the need for a poling field [12]. The physics principle for the ESA films to exhibit even-order NLO effects without poling is because the cationic and anionic molecules are aligned in a polar manner during the alternating adsorption processes due to Coulomb force occurring between cations and anions. We report here detailed studies of the electrostatic self-assembly technique for the creation of noncentrosymmetric thin films for NLO applications without the need for additional processing such as electric-field poling or chemical reaction. We also present an extended theory that may be used to determine the NLO coefficients of such films. 
Figure 1 shows the basic concept of the ESA process. The substrate surface has been thoroughly cleaned and charged through chemical processing. The negatively charged substrate is then dipped into a polycation solution to form a monolayer of positively charged molecules at the surface. After rinsing with ultra-pure water to remove any loose molecules that are not ionically bonded to the surface, the coated substrate is then dipped into a polyanion solution. Subsequently, a monolayer of negative molecules is added to form the outermost surface layer. By alternately dipping the substrate into polycation and polyanion solutions, a multilayer thin film is grown as shown in the figure. For the work reported here, ITO-coated glass substrates were cleaned thoroughly using a solution of acetone and isopropyl alcohol, then rinsed extensively with ultrapure water.

Figure 1. The electrostatically self-assembled (ESA) process used to fabricate thin films: (a) charged substrate and assembly of first polyelectrolyte monolayer; (b) charged substrate, the first monolayer and assembly of second polyelectrolyte monolayer; (c) charged substrate, the first bilayer and assembly of third polyelectrolyte monolayer.

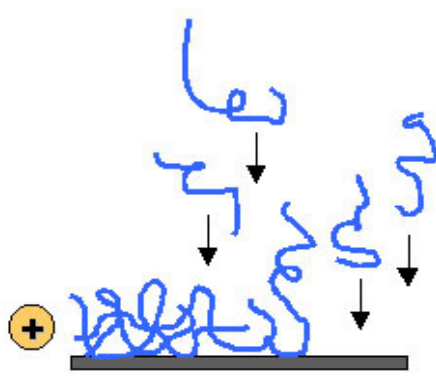

(a)

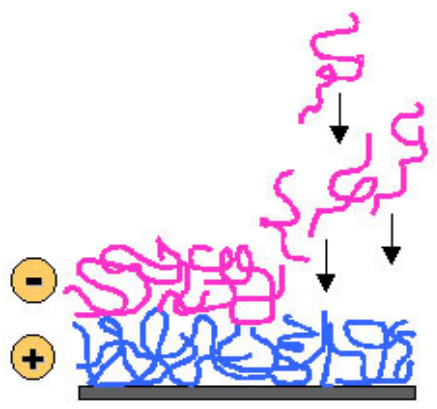

(b)

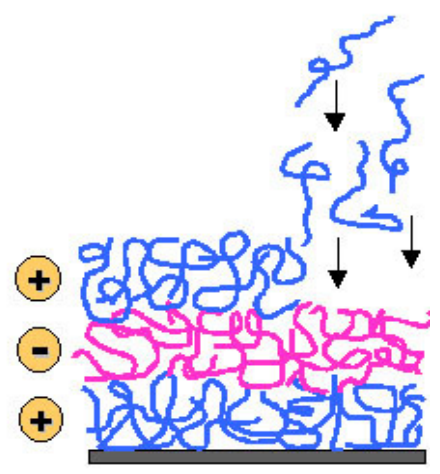

(c)

For the fabrication of samples used in these measurements, poly S-119 and poly (diallyl dimethyl ammonium chloride) (PDDA) were purchased from Sigma and Aldrich, respectively. Figure 2 shows the chemical structures of poly S-119 and PDDA. The substrate was first immersed in a cationic 1\% PDDA (v/v) aqueous solution for $1 \mathrm{~min}$, then rinsed extensively in ultrapure water, forming a monolayer of PDDA molecules. Subsequent immersion in an anionic aqueous solution of poly S-119 with a concentration of $2 \mathrm{mg} / \mathrm{mL}$ for $1 \mathrm{~min}$, again followed by thorough rinsing in ultrapure water, produced a uniform poly S-119 monolayer on the top film surface. Additional bilayers were added by repetition of this alternating two-step process, until the desired thickness was achieved. Uniform poly S-119 films have been obtained with an average bilayer thickness on the order of $2 \mathrm{~nm}$ based on ellipsometer measurement data. Figure 3 shows UV-vis spectroscopy (Hitachi U-2001) data for a sample with a thickness of 200 bilayers. It can be seen that the absorbance peak of the film is located at approximately $480 \mathrm{~nm}$ and the film is approximately transparent for wavelengths greater than $580 \mathrm{~nm}$. 
Figure 2. The structures of poly S-119 and poly(diallyl dimethyl ammonium chloride) (PDDA).

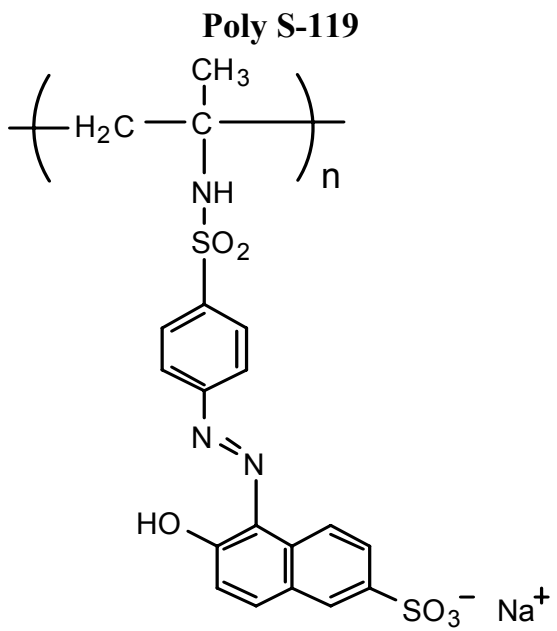

PDDA

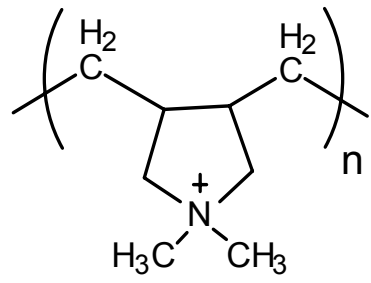

Figure 3. Absorbance versus wavelength. It can be seen that the film is transparent at wavelengths greater than $580 \mathrm{~nm}$.

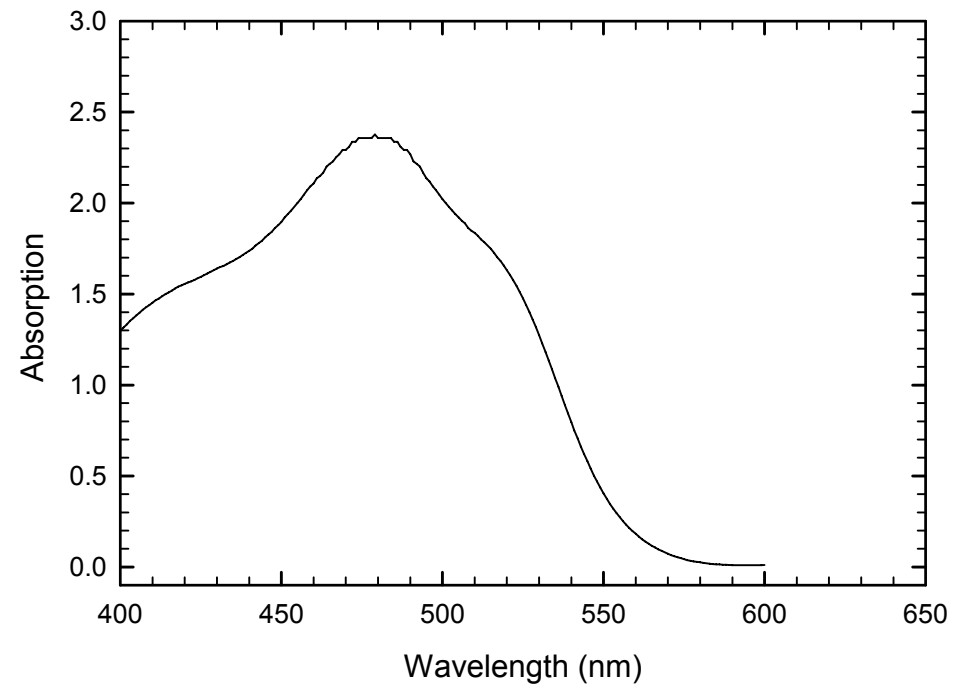

\section{Theory}

Theoretical and experimental investigations of SH generation in thin films have been proposed and developed [13-20]. However, in most of these cases, the films were fabricated by the spin-coating technique and the sample cell includes a film sandwiched between two glass plates. In our case, the film was deposited instead only on one side of a glass substrate. We therefore need to seek special solutions to Maxwell's equations as boundary conditions. A more detailed related evaluation process can be found in [20]. Figure 4 shows the incident beam geometry, where the film was deposited on one side of the glass substrate using the ESA technique. The film material has $\mathrm{C}_{\infty v}$ symmetry, similar to field-poled spin-coated films and Langmuir-Blodgett films [21]. When Kleiman symmetry [22] holds, $\chi_{311}$ and $\chi_{333}$ are only the non-zero components of the second-harmonic susceptibilities. 
Figure 4. The structure of the sample and the incident geometry in our experiments used: G, glass substrate; F, film; p-p, both SH and incident beam are p-polarized; s-p, incident beam is s-polarized and $\mathrm{SH}$ is p-polarized.

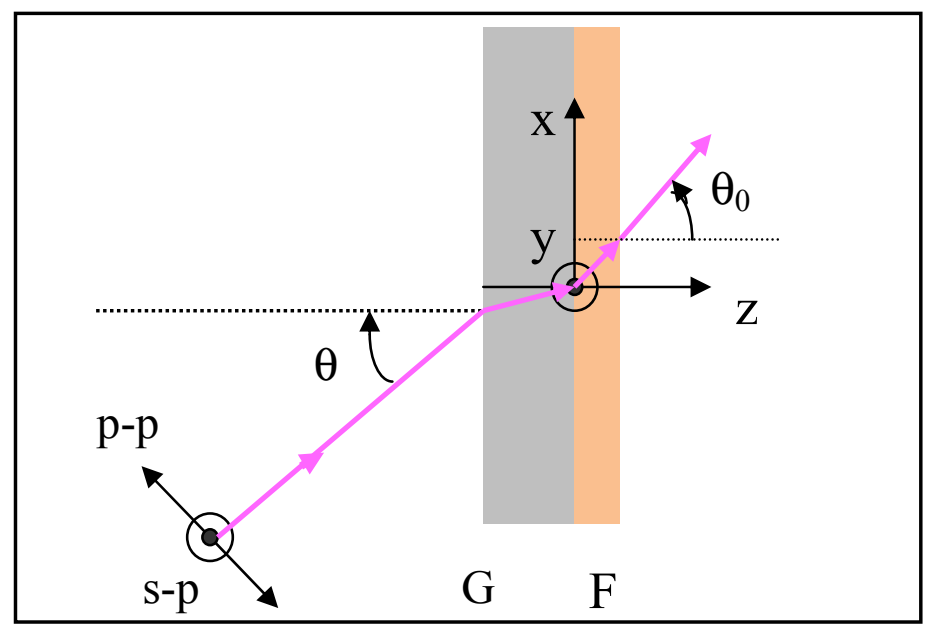

Using field boundary conditions at $\mathrm{z}=0$ and $\mathrm{z}=1$ ( 1 is the thickness of the film) and neglecting the refractive index dispersion of air, after some manipulations, the intensity $\mathrm{I}_{2 \omega}$ of the SH wave in air can be written as [20]

$$
I_{2 \omega}=\frac{2 \chi_{311}^{2} t_{\omega}^{4} T_{2 \omega} I_{\omega}^{2} \sin ^{2} \psi}{\sqrt{\varepsilon_{0} / \mu_{0}} \varepsilon_{0}^{2}\left(n_{\omega}^{2}-n_{2 \omega}^{2}\right)},
$$

where

$$
\begin{gathered}
T_{2 \omega}=2 F\left[n_{2 \omega} / n_{2 \omega}^{o}\right]^{2} \cos \theta_{2 \omega}^{f}\left(f n_{2 \omega} \cos \theta_{2 \omega}^{f} /\left(n_{2 \omega}^{o}\right)^{2}+g\right) / h^{\prime 2}, \\
\psi=(\omega / c) l\left(n_{\omega} \cos \theta_{\omega}^{f}-n_{2 \omega} \cos \theta_{2 \omega}^{f}\right), \\
h^{\prime}=\cos \theta_{0}+n_{2 \omega} \cos \theta_{2 \omega}^{f} /\left(n_{2 \omega}^{o}\right)^{2}, \\
F=\left(f \cos \theta_{2 \omega}^{g}+n_{2 \omega}^{g} g\right) /\left[n_{2 \omega} h\right], \\
g=p_{x}\left(n_{2 \omega} / n_{2 \omega}^{o}\right)^{2}-\left(p_{x} \sin \theta_{\omega}^{f}+p_{z} \cos \theta_{\omega}^{f}\right)\left[n_{\omega} n_{2 \omega} / n_{2 \omega}^{o} n_{2 \omega}^{e}\right]^{2} \sin \theta_{\omega}^{f}, \\
h=\cos \theta_{2 \omega}^{g}+n_{2 \omega}^{g} n_{2 \omega} \cos \theta_{2 \omega}^{f} /\left(n_{2 \omega}^{o}\right)^{2}, \\
n_{2 \omega}=\left[\left(\cos \theta_{\omega}^{f} / n_{2 \omega}^{o}\right)^{2}+\left(\sin \theta_{\omega}^{f} / n_{2 \omega}^{e}\right)^{2}\right]^{-1 / 2},
\end{gathered}
$$

where $\mathrm{n}_{2 \omega}^{\mathrm{g}}$ is the refractive index of the glass at $2 \omega, \theta^{\mathrm{g}}{ }_{2 \omega}$ is the refractive angle of the second-harmonic signal in the glass, $\mathrm{n}_{\omega}$ and $\mathrm{n}_{2 \omega}$ are refractive indices of the film at the fundamental and harmonic frequencies, $\mathrm{n}^{\circ}{ }_{\omega}, \mathrm{n}^{\mathrm{e}}{ }_{\omega}, \mathrm{n}^{\circ}{ }_{2 \omega}$, and $\mathrm{n}_{2 \omega}^{\mathrm{e}}$ are ordinary and extraordinary refractive indices of the film at fundamental and harmonic frequencies, respectively, and $t_{\omega}$ is the product of the transmission coefficients at the air-glass and glass-film interfaces. For the incident s-polarized component

$$
t_{\omega}=\frac{4 n_{\omega}^{g} \cos \theta \cos \theta_{\omega}^{g}}{\left(\cos \theta+n_{\omega}^{g} \cos \theta_{\omega}^{g}\right)\left(n_{\omega}^{o} \cos \theta_{\omega}^{f}+n_{\omega}^{g} \cos \theta_{\omega}^{g}\right)},
$$


and for the incident p-polarized component

$$
t_{\omega}=\frac{4 n_{\omega}^{g} \cos \theta \cos \theta_{\omega}^{g}}{\left(n_{\omega}^{g} \cos \theta+\cos \theta_{\omega}^{g}\right)\left[n_{\omega}^{g}\left(n_{\omega} / n_{\omega}^{o}\right)^{2} \cos \theta_{\omega}^{f}+n_{\omega} \cos \theta_{\omega}^{g}\right]},
$$

here $n^{\mathrm{g}}{ }_{\omega}$ is the refractive index of the glass at $\omega, \theta^{\mathrm{g}}{ }_{\omega}$ is the refractive angle in the glass and $\theta_{\omega}^{\mathrm{f}}$ is the refractive angle of the fundamental beam at the glass-film interface given by Snell's law

$$
n_{\omega} \sin \theta_{\omega}^{f}=n_{\omega}^{g} \sin \theta_{\omega}^{g}=\sin \theta
$$

For s-polarized incidence

$$
\mathbf{p}_{2 \omega}=\left(p_{x}, 0, p_{y}\right)=(0,0,1)
$$

and for p-polarized incidence

$$
\mathbf{p}_{2 \omega}=\left(p_{x}, 0, p_{y}\right)=\left(n_{\omega}\right)^{4}\left[-\sin \left(2 \theta_{\omega}^{f}\right) /\left(n_{\omega}^{o} n_{\omega}^{e}\right)^{2}, 0, \cos ^{2} \theta_{\omega}^{f} /\left(n_{\omega}^{o}\right)^{4}+r \sin ^{2} \theta_{\omega}^{f} /\left(n_{\omega}^{e}\right)^{4}\right],
$$

where $\mathbf{p}_{\mathbf{2} \omega}$ is the second-order nonlinear polarization, the detailed calculations for the expressions of Equations (13) and (14) can be found in [20]. $r=\chi_{333} / \chi_{311}$. Note that $T_{2 \omega}$ includes $r=\chi_{333} / \chi_{311}$ only for p-polarized incidence while $I_{2 \omega}$ only includes $\chi_{311}$ for s-polarized incidence. Therefore, if one can experimentally measure $I_{2 \omega}$ as a function of the incident angle for the p-polarized incidence, one should be able to obtain $r$ using a computational fitting method. Moreover, if one can obtain $\chi_{311}$ from the s-polarized incidence, $\chi_{333}$ and $\chi_{311}$ can be determined.

Because absolute measurements of fundamental and $\mathrm{SH}$ intensities are always difficult, for more accurate determination of nonlinear optical coefficients of the thin films, researchers now often use inorganic crystals as reference materials since careful measurements for these crystals have been made. In most cases, quartz is widely used as a reference material [24-26]. To determine $\chi_{311}$ for the s-polarized incidence, we used a Y-cut quartz platelet with a thickness of $1 \mathrm{~mm}$ as a reference. The SH intensity from this reference was measured using the same fundamental intensity as that used for our sample films. The rotation axis is the x-axis. The fundamental beam polarized along the $\mathrm{x}$-axis was incident upon the quartz along the Y direction. The SH intensity from the quartz platelet is [19]

$$
I_{2 \omega}^{q}=\frac{2 \chi_{111}^{2} t_{\omega}^{4} T_{2 \omega}^{\prime} I_{\omega}^{2} \sin ^{2} \psi^{\prime}}{\sqrt{\varepsilon_{0} / \mu_{0}} \varepsilon_{0}^{2}\left(n_{\omega}^{\prime 2}-n_{2 \omega}^{\prime 2}\right)},
$$

where $\chi_{111}$ is one of the nonlinear optical coefficients of quartz, $n^{\prime}{ }_{\omega}$ and $n^{\prime}{ }_{2 \omega}$ are refractive indices of the quartz at the fundamental and the $\mathrm{SH}$ wavelengths, respectively, and

$$
\begin{gathered}
t_{\omega}^{\prime}=\frac{2 \cos \theta^{\prime}}{\left(n_{\omega}^{\prime}{ }_{\omega} \cos \theta_{\theta}^{q}+\cos \theta^{\prime}\right)}, \\
T^{\prime}{ }_{2 \omega}=2 n_{2 \omega}^{\prime} \cos \theta_{2 \omega}^{q} \frac{\left(\cos \theta^{\prime}+n_{\omega}^{\prime} \cos \theta_{\omega}^{q}\right)\left(n_{\omega}^{\prime} \cos \theta_{\omega}^{q}+n_{2 \omega}^{\prime} \cos \theta_{2 \omega}^{q}\right)}{\left(n^{\prime}{ }_{2 \omega} \cos \theta_{2 \omega}^{q}+\cos \theta_{\omega}^{q}\right)^{3}}, \\
\psi^{\prime}=\frac{2 \pi l^{\prime}}{\lambda}\left(n_{\omega}^{\prime}{ }_{\omega} \cos \theta_{\omega}^{q}-n^{\prime}{ }_{2 \omega} \cos \theta_{2 \omega}^{q}\right),
\end{gathered}
$$

where $l^{\prime}$ is the thickness of the quartz platelet, $\theta^{\prime}$ is the incident angle, and $\theta^{\mathrm{q}}{ }_{\omega}$ and $\theta^{\mathrm{q}}{ }_{2 \omega}$ are the refractive angles at the fundamental and the $\mathrm{SH}$ wavelengths, respectively. Using Equations (1) and (15), one has 


$$
\frac{I_{2 \omega}}{I^{\prime}{ }_{2 \omega}}=\left(\frac{\chi_{311}}{\chi_{111}}\right)^{2} \cdot\left(\frac{t_{\omega}}{t^{\prime}{ }_{\omega}}\right)^{4} \cdot\left(\frac{T_{2 \omega}^{\prime}}{T_{2 \omega}}\right) \cdot\left(\frac{n_{\omega}^{\prime 2}-n_{2 \omega}^{\prime 2}}{n_{\omega}^{2}-n_{2 \omega}^{2}} \frac{\sin \psi}{\sin \psi^{\prime}}\right)^{2}
$$

One can see that $\chi_{311}$ now can be calculated using $\chi_{111}$ and experimentally measured values for $\mathrm{I}_{2 \omega}$ and I' $2 \omega$.

\section{Experiment}

Experiments were performed using a Coherent femto-second ultrafast laser system as shown in Figure 5. A pump laser (V-10) operating at $532 \mathrm{~nm}$ with an output power of $10 \mathrm{~W}$ pumps a Ti-Sapphire laser (Mira-900F). The output wavelength of the Ti-Sapphire laser was adjusted to $840 \mathrm{~nm}$ to pump a ring-cavity optical parametric oscillator (OPO). The pumped OPO (pulse width 200 fs and repetition rate $75.86 \mathrm{MHz}$ ) operated at $1,200 \mathrm{~nm}$ with an output power of approximately $300 \mathrm{~mW}$, and a beam diameter of $5 \mathrm{~mm}$ was used as the fundamental wavelength. A half-wave plate (HF) and a Glan-Thomson polarizer (P1) were used to control the polarization and power of the fundamental incident beam. Two interference band-pass filters (F1, central wavelength 1,200 nm and FWHM $11.2 \mathrm{~nm}$ ) were introduced before the sample to block the interfering SH wavelength that was possibly created in the laser system. The sample was mounted on a rotation stage (angle accuracy $\pm 1^{\circ}$ ). After the sample, an interference band-pass filter (F2, central wavelength $600 \mathrm{~nm}$ and FWHM $10.1 \mathrm{~nm}$ ) was used to remove the fundamental beam power. $\mathrm{P} 2$ is another polarizer used to analyze the polarization of the SH signal. Then, the created SH signal went through a monochromator (Oriel Corner Stone 1/4 M) that was adjusted at $600 \mathrm{~nm}$, and was detected by a PMT (Oriel 77348) and a lock-in amplifier (Stanford Research System SRS-850). A storage oscilloscope (TDS-430A) was also used to monitor the SH signal.

Figure 5. Experimental setup to measure SH generation: PL, pump laser; FL, femtosecond laser pumped by the pump laser; OPO: optical parameter oscillator pumped by the femtosecond laser; $\mathrm{C}$ : chopper to provide a reference signal to the lock-in amplifier; HF, half-wave plate at 1,200 nm; P1: polarizer; F's: interference bandpass filters; S: sample; P2: analyzer; M: monochromator; PMT: photomultiplier; OSC: oscilloscope; LA: Lock-in amplifier.

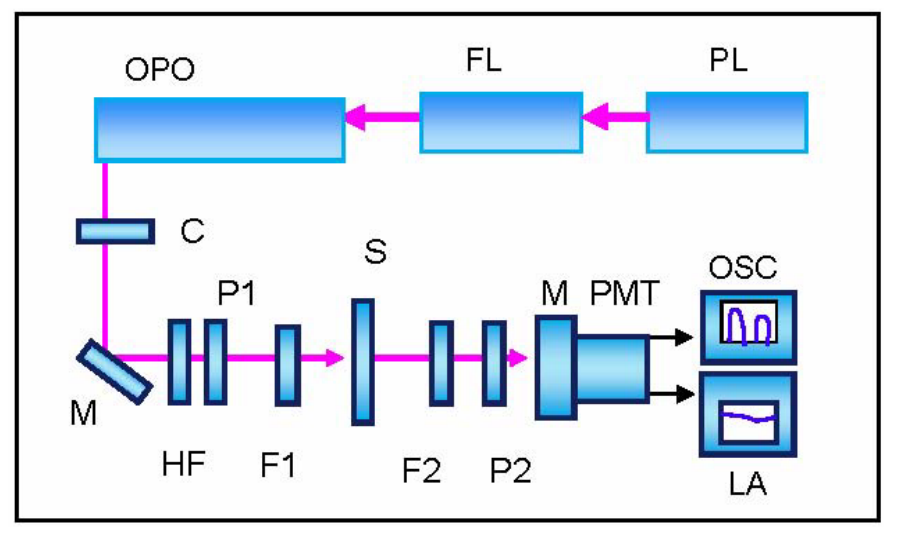

Using the p-polarized fundamental incidence, we measured the p-polarized SH intensity (p-p configuration) as a function of the incident angle in the poly S-119/PDDA film. The measurements show that the maximum SH intensity occurred at $\theta=60^{\circ}$. As mentioned above, due to the difficulty of 
accurately measuring absolute intensities of both fundamental and $\mathrm{SH}$ waves, to obtain $\mathrm{d}_{\mathrm{r}}$ more accurately, we have used normalized signal to remove $\mathrm{I}^{2}{ }_{\omega}$ from Equation (1). Because the maximum SH intensity was obtained at $\theta=60^{\circ}$, we treated the SH intensity at $\theta=60^{\circ}$ as unity to normalize the SH intensities at other incident angles.

\section{Results and Discussion}

Figure 6 shows the intensity of the SH signal as a function of power at the fundamental wavelength at an incident angle of $60^{\circ}$. Symbols correspond to the measured results and the solid-curve indicates a best fit to the form $I_{2 \omega}=a\left(I_{\omega}\right)^{b}$, where $I_{2 \omega}$ and $I_{\omega}$ are the second-harmonic and fundamental intensities, respectively. The fits yields a value of $b=1.98$ and $a=0.053$, which is in good agreement with the quadratic dependence on fundamental intensity.

Figure 6. Dependence of the $\mathrm{SH}$ intensity at $600 \mathrm{~nm}$ on the fundamental intensity at $1,200 \mathrm{~nm}$ in the $\mathrm{p}-\mathrm{p}$ incidence configuration.

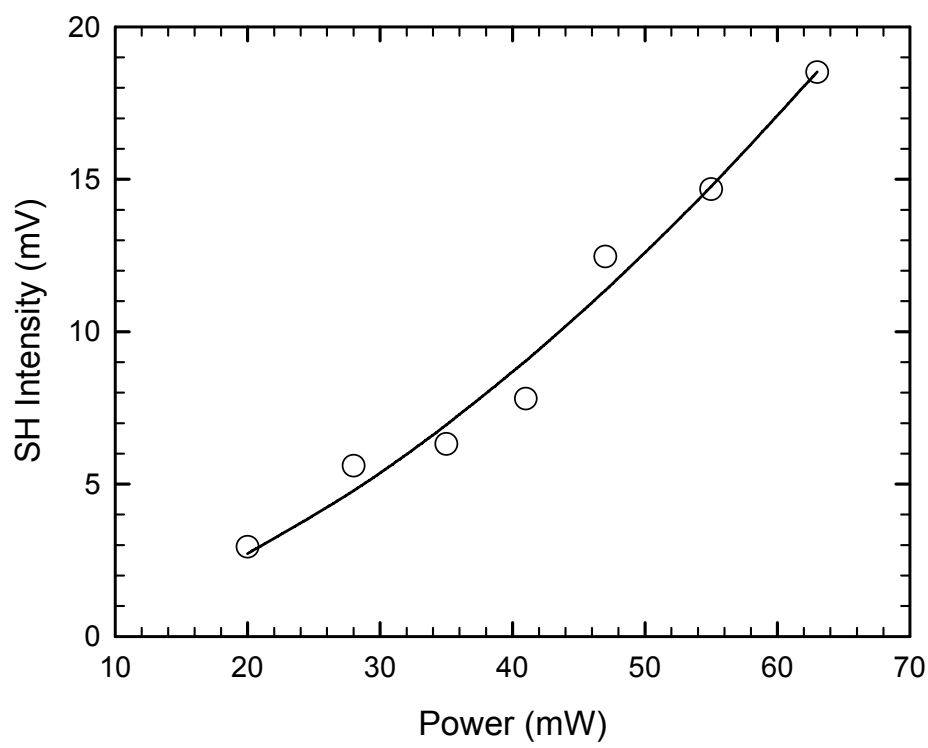

Figure 7 shows the normalized SH intensity (p-p) as a function of the incident angle. The symbols and solid-curve correspond to the measured and fitted results, respectively. The fundamental laser power used is $75 \mathrm{~mW}$. Using the fitted results of Equation (1), as an approximation, let us take $\mathrm{n}_{2 \omega}=\mathrm{n}_{2 \omega}^{\circ}=\mathrm{n}_{2 \omega}^{\mathrm{e}}=1.639, \mathrm{n}_{\omega}=\mathrm{n}^{\circ}{ }_{\omega}=\mathrm{n}_{\omega}^{\mathrm{e}}=1.488$, refractive indices of the optical glass $\mathrm{n}^{\mathrm{g}}{ }_{\omega}=1.513$, $\mathrm{n}^{\mathrm{g}}{ }_{2 \omega}=1.516$ [29], and neglect the refractive index dispersion of air. Then, the fitted result gives $\mathrm{r}=\chi_{333} / \chi_{311}=9.07 \pm 0.45$ after using the normalization method to eliminate $\mathrm{I}_{2 \omega}^{2}$ from Equation (1).

To separate $\chi_{333}$ and $\chi_{311}$, as mentioned above, we have to compute $\chi_{311}$ first by using the s-polarized fundamental beam to obtain the p-polarized SH intensity from the film in comparison with that from the quartz platelet under the same conditions. We used the s-polarized fundamental beam to measure the p-polarized SH intensity as a function of the incident angle from the film (s-p configuration). Because only $\chi_{311}$ can contribute to the SH intensity of this polarization configuration, the SH signal is much weaker than that of the p-p configuration. To obtain a more accurate value of $\chi_{311}$, we measured the SH signal as a function of the incident angle over a wide range $\left(10^{\circ}-70^{\circ}\right)$ instead of only at one point. For comparison, the Y-cut quartz sample was measured under the same 
intensity of the fundamental beam. The quartz sample was rotated to the maximum SH orientation at near normal incidence. The rotation axis is the X-axis and the fundamental beam polarized in the $\mathrm{X}$-axis was incident upon the quartz along $\mathrm{Y}$ direction (corresponding to the effective second-order susceptibility $\left.\chi_{111}\right)$. Then, we treat the signal of the quartz sample as unity to normalize the SH signal from the film. Using Equation (19) to fit the normalized result, one can obtain $\chi_{311}$. Figure 8 shows the normalized SH signal. The symbols and solid-curve indicate the measured and fitted results, respectively. Taking $\chi_{111}=0.68 \mathrm{pm} / \mathrm{V}$ [25-27], $\mathrm{n}_{2 \omega}=1.547$ and $\mathrm{n}_{\omega}{ }_{\omega}=1.534$ [19], we then obtained $\chi_{311}=0.68 \pm 0.02 \mathrm{pm} / \mathrm{V}$. Using $\chi_{333} / \chi_{311}=9.07 \pm 0.45$ yields $\chi_{333}=6.17 \pm 0.18 \mathrm{pm} / \mathrm{V}$.

Figure 7. Normalized $\mathrm{SH}$ intensity as a function of the incident angle in the $p-p$ configuration. The symbols and solid-curve correspond to the measured and fitted results, respectively.

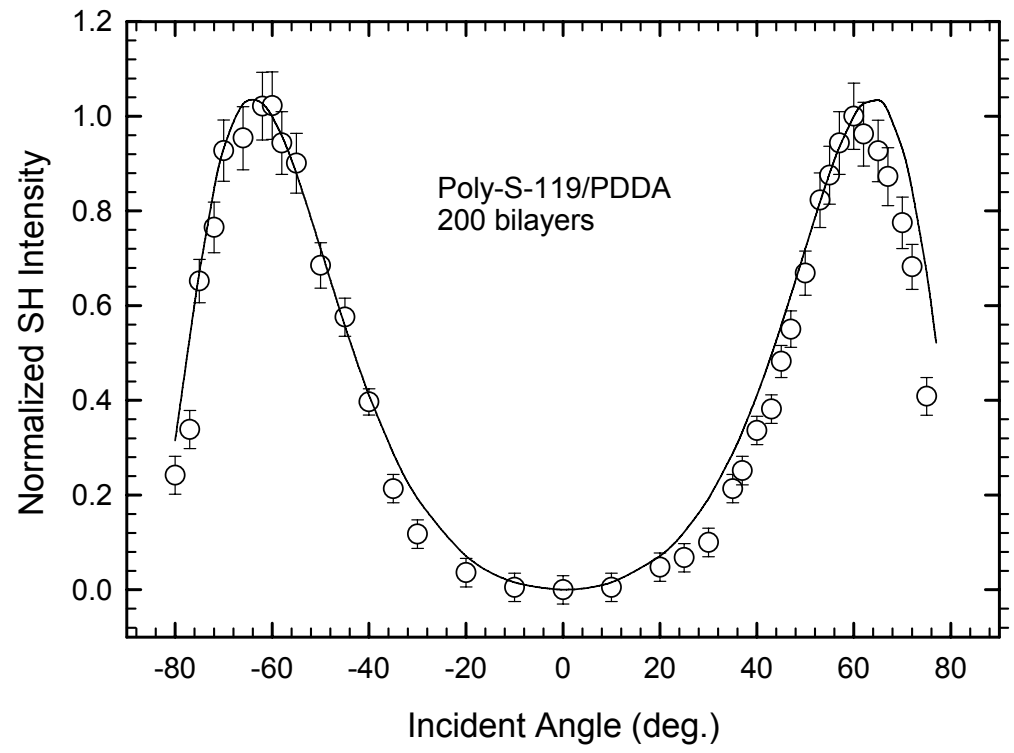

Figure 8. Normalized $\mathrm{SH}$ intensity as a function of the incident angle in the $s-p$ configuration. The symbols and solid-curve correspond to the measured and fitted results, respectively.

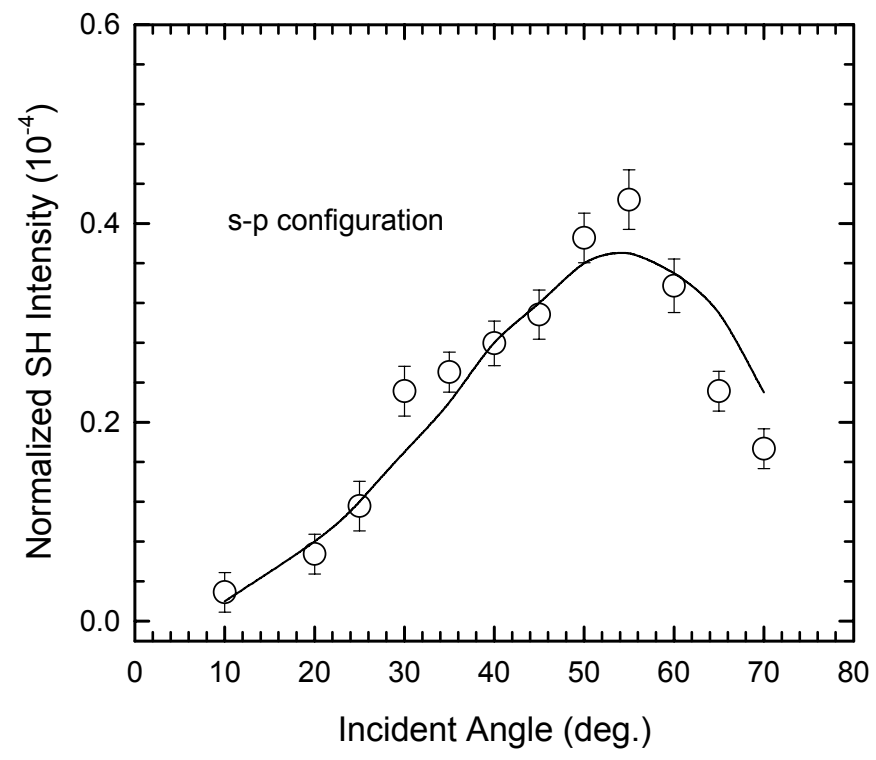




\section{Molecular Orientation}

Second-harmonic generation is an important tool to determine molecular dipole tilt angle [30], however, detailed analysis for the orientation angle distribution of the poly s-119 dipoles is beyond the scope of this work. We use an approximation model of one-dimensional molecular system to concentrate on the determination of mean tilt angle of the poly s-119 molecules. In the approximation model of a one-dimensional molecular system, assuming that $\beta_{z z z}$ is the only dominant component of the second-order optical susceptibility, $\chi_{333}$ and $\chi_{311}$ can be written as $[14,17,28]$

$$
\begin{aligned}
& \chi_{333}=N \beta_{z z z}^{*}\left(\frac{3}{5}\left\langle P_{1}\right\rangle+\frac{2}{5}\left\langle P_{3}\right\rangle\right) \\
& \chi_{311}=N \beta_{z z z}^{*}\left(\frac{1}{5}\left\langle P_{1}\right\rangle-\frac{1}{5}\left\langle P_{3}\right\rangle\right),
\end{aligned}
$$

where $\left\langle\mathrm{P}_{1}>\right.$ and $\left\langle\mathrm{P}_{3}\right\rangle$ are order parameters related to the Legendre polynomials, $\mathrm{N}$ is the number

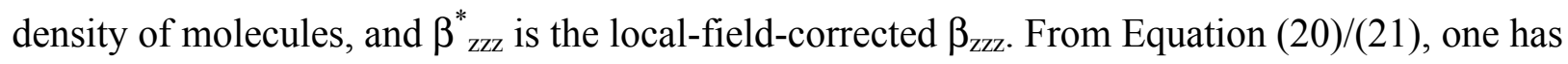

$$
\frac{\left\langle P_{3}\right\rangle}{\left\langle P_{1}\right\rangle}=\frac{r-3}{r+2},
$$

where $r=\chi_{333} / \chi_{311}$. From Equations (20) and (21), one can see that $\left\langle\mathrm{P}_{3}\right\rangle=0$ gives the minimum $\mathrm{r}=3$. This corresponds to isotropic materials [14,18]. With the orientation of chromophore dipoles increasing, $<\mathrm{P}_{3}>$ increases. As a result, the value of $\mathrm{r}$ is increased as well. In an extreme case when the order parameters are unity, one has $\mathrm{r} \sim \infty$. In our case, the computer fit gives $r=9.07 \pm 0.45$, which yields $\left\langle\mathrm{P}_{3}>\right|<\mathrm{P}_{1}>\sim 0.55$. This indicates that the molecular orientation in our films has been significantly improved compared to isotropic materials. Figure 9 shows the average tilt angle $\delta$ of poly S-119 molecular dipoles from the substrate normal. The poly S-119 molecule has been simplified to the linear molecule assumption, the average tilt angle $\delta$ is determined by

$$
\chi_{333} / \chi_{311}=2 \cot ^{2} \delta
$$

and the fitted result of $\chi_{333} / \chi_{311}=9.07 \pm 0.45$ yields $\delta=25.2^{\circ} \pm 0.8^{\circ}$.

Figure 9. The average tilt angle of the poly S-119 molecular dipoles from the substrate normal. The poly S-119 molecule has been simplified by a linear model approximation [28]. S: substrate; $\delta$ : average tilt angle.

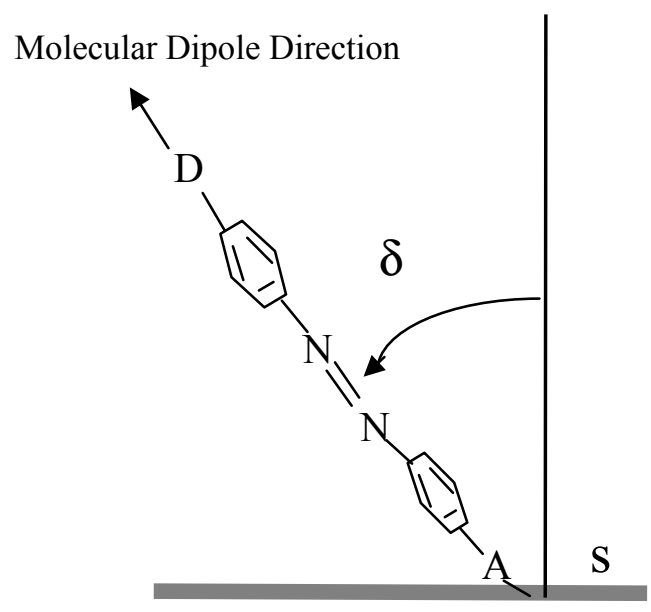




\section{Conclusions}

We have shown that thin films fabricated using the ESA technique can spontaneously assemble into a noncentrosymmetric structure with substantial second-harmonic generation effect, without the need of external electric field poling. Devices made using this approach may have potential applications in electro-optic and frequency doubling waveguide devices [23-32]. A method to determine the second-order optical susceptibilities of thin films has been presented. The average orientation of the molecular dipoles has been discussed and the ratio of two important order parameters to describe the molecular orientation has been given. The results indicate that the orientation of the chromophore dipoles has been significantly improved over that of isotropic materials.

\section{Acknowledgements}

The authors would like to thank Fajian Zhang, You-Xiong Wang, Richard O. Claus and David Wood at NanoSonic Incorporated for their assistance in fabrication of these films.

\section{References}

1. Meredith, G.R.; Van Dusen, J.; Williams, D.J. Optical and nonlinear optical characterization of molecularly doped thermotropic liquid crystalline polymers. Macromolecules 1982, 15, 1385-1389.

2. Garito, A.F; Singer, K.D. Organic crystals and polymers-A new class of nonlinear optical materials. Laser Focus 1982, 80, 59-65.

3. Lindsay, G.A.; Singer, K.D. Polymers for second-order nonlinear optics. ACS Symp. Ser. 1995, doi: 10.1021/bk-1995-0601.fw001.

4. Singer, K.D.; Kuzyk, M.G.; Holland, W.R.; Sohn, J.E.; Lalama, S.J. Electro-optic phase modulation and optical second-harmonic generation in corona-poled polymer films. Appl. Phys. Lett. 1988, 53, 1800-1822.

5. Kippelen, K.; Meerholz, S.K.; Peyhambarian, N. Birefringence, Pockels, and Kerr effects in photorefractive polymer. Appl. Phys. Lett. 1998, 68, 1748-1750.

6. Moerner, W.E.; Silence, S.M; Hache, F.; Bjorklund, G.C. Orientationally enhanced photorefractive effect in polymers. J. Opt. Soc. Am. B 1994, 11, 320-330.

7. Winiarz, J.G.; Zhang, L.; Lal, M.; Friend, C.S.; Prasad, P.N. Observation of the photorefractive effect in a hybrid organic-inorganic nanocomposite. J. Am. Chem. Soc. 1999, 121, 5287-5295.

8. Decher, G.; Hong, J.D.; Schmitt, J. Buildup of ultrathin multilayer films by a self-assembly process: III. Consecutively alternating adsorption of anionic and cationic polyelectrolytes on charged surfaces. Thin Solid Films 1992, 210-211, 831-835.

9. Lvov, Y.; Yamada, S.; Kunitake, T. Nonlinear optical effects in layer-by-layer alternate films of polycations and an azobenzene-containing polyanion. Thin Solid Films 1997, 300, 107-112.

10. Katz, H.E.; Wilson W.L.; Scheller, G. Chromophore structure, second harmonic generation, and orientation order in zirconium phosphonate/phosphate self-assembed multilayers. J. Am. Chem. Soc. 1994, 116, 6636-6640. 
11. Tojima, A.; Manaka, T.; Iwamoto, M.; Yang, O.; Zhong-Can, O. Detection of phase transition of monolayers at the air-water interface by compression using Maxwell displacement current and optical second harmonic generation. J. Chem. Phys. 2003, 118, 5640-5649.

12. Zhang, L.; Zhang, F.; Wang, Y.; Claus, R.O. Linear electro-optic tensor ration determination and quadratic electro-optic modulation of electrostatically self-assembled CdSe quantum dot fllms. J. Chem. Phys. 2002, 116, 6297-6304.

13. Bloembergen, N.; Pershan, P.S. Light waves at the boundary of nonlinear media. Phys. Rev. 1962, $128,606-622$.

14. Singer, K.D.; Kuzyk, M.G.; Sohn, J.E. Second-order nonlinear-optical processes in orientationally ordered materials: Relationship between molecular and macroscopic properties. J. Opt. Soc. Am. B 1987, 4, 968-976.

15. Singer, K.D.; Garito, A.F. Measurements of molecular second order optical susceptibilities using dc induced second harmonic generation. J. Chem. Phys. 1981, 75, 3572-3581.

16. Lalama, S.J.; Garito, A.F. Origin of the nonlinear second-order optical susceptibilities of organic systems. Phys. Rev. A 1979, 20, 1179-1194.

17. Huang, J.Y.; Chen, Z.; Lewis, A. Second-harmonic generation in purple membrane-poly(vinyl alcohol) films: Probing the dipolar characteristics of the bacteriorhodopsin chromophore in bR570 and M412. J. Phys. Chem. 1989, 93, 3314-3320.

18. Kuzyk, M.G.; Singer, K.D.; Zahn, H.E.; King, L.A. Second-order nonlinear-optical tensor properties of poled films under stress. J. Opt. Soc. Am. 1989, 6, 742-752.

19. Jerphagnon, J.; Kurtz, S.K. Maker fringes: A detailed comparison of theory and experiment for isotropic and uniaxial crystals. J. Appl. Phys. 1970, 41, 1667-1685.

20. Okamoto, N.; Hirano, Y.; Sugihara, O. Precise estimation of nonlinear-optical coefficients for anisotropic nonlinear films with Coov symmetry. J. Opt. Soc. Am. B 1992, 9, 2083-2087.

21. Howarth, V.A.; Asai, N.; Kishii, N.; Fujiwara, I. Optical second harmonic generation in Langmuir-Blodgett films of $n$-docosyl-2-methyl-4-nitroaniline. Appl. Phys. Lett. 1992, 61, 1616-1618.

22. Kleinman, D.A. Theory of second harmonic generation of light. Phys. Rev. 1962, 128, 1761-1775.

23. Prasad, P.N.; Williams, D.J. Introduction to Nonlinear Optical Effects in Molecules and Polymers; Wiley: New York, NY, USA, 1991.

24. Lu, H.A.; Wills, L.A.; Wessels, B.W.; Lin, W.P.; Zhang, T.G.; Wong, G.K.; Neumayer, T.J. Marks, T.H. Second-harmonic generation of poled $\mathrm{BaTiO}_{3}$ thin films. Appl. Phys. Lett. 1993, 62, 1314-1316.

25. Bihari, B.; Kumar, J.; Stauf, G.T.; Bushkirk, P.C.V.; Hwang, C.S. Investigation of barium titanate thin films on $\mathrm{MgO}$ substrates by second-harmonic generation. J. Appl. Phys. 1994, 76, 1169-1174.

26. Okada, T.; Nakata, Y.; Kaibara, H.; Maeda, M. Second-harmonic generation in pulsed-laser deposited $\mathrm{BaTiO}_{3}$ thin films. Jpn. J. Appl. Phys. 1995, 34, L1536-L1539.

27. Kaye G.W.C.; Laby, T.H. Tables of Physical and Chemical Constants and Some Mathematical Functions; Longman: Harlow, UK, 1951. 
28. Rasing, T.; Shen, Y.R.; Kim, M.W.; Valint, P.; Bock, J. Orientation of surfactant molecules at a liquid-air interface measured by optical second-harmonic generation. Phys. Rev. A 1985, 31, 537-539.

29. Li, D.Q; Ratner, M.A.; Marks, T.J. Chromophoric self-assembled multilayers. Organic superlattice approaches to thin-film nonlinear optical materials. J. Am. Chem. Soc. 1990, 112, 7389-7390.

30. Simpson, G.J.; Rowlen, K.L. An SHG magic angle: Dependence of second harmonic generation orientation measurements on the width of the orientation distribution. J. Am. Chem. Soc. 1999, $121,2635-2636$.

31. Otomo, A.; Stegeman, G.I.; Horsthuis, W.H.G.; Möhlmann, G.R. Quasi-phase matched surface emitting second harmonic generation in poled polymer waveguides. Appl. Phys. Lett. 1996, 68, 3683-3685.

32. Shi, Y.; Zhang, C.; Zhang, H.; Bechtel, J.; Dalton, L.R.; Robinson, B.H.; Steier, W.H. Low (Sub-1-volt) halfwave voltage polymeric electro-optic modulators achieved by controlling chromophore shape. Science 2000, 288, 119-122.

(C) 2011 by the authors; licensee MDPI, Basel, Switzerland. This article is an open access article distributed under the terms and conditions of the Creative Commons Attribution license (http://creativecommons.org/licenses/by/3.0/). 